\title{
Evaluation der DEGUM-Mammasonografiekurse nach objektivierbaren Kriterien
}

\section{Evaluation of the Nationwide DEGUM Breast Ultrasound Training Program}

Authors

Affiliations
R. Würstlein ${ }^{1}$, F. Degenhardt² ${ }^{2}$, V. Duda ${ }^{3}$, H. Madjar ${ }^{4}$, E. Merz ${ }^{5}$, A. Mundinger ${ }^{6}$, R. Ohlinger ${ }^{7}$, U. Peisker ${ }^{8}$, R. Schulz-Wendtland ${ }^{9}$, M. Warm ${ }^{10}$, M. Hahn ${ }^{11}$

Die Institutsangaben sind am Ende des Beitrags gelistet.

Key words
breast
QA/QC
training
education

received $\quad 8.8 .2013$

accepted 28.1.2014

Bibliography

DOI http://dx.doi.org/

10.1055/s-0034-1366088

Published online: February 21, 2014

Ultraschall in Med 2014; 35 :

345-349 @ Georg Thieme

Verlag KG Stuttgart · New York .

ISSN 0172-4614

\section{Correspondence}

\section{PD Dr. Markus Hahn}

Frauenklinik, Universität

Tübingen

Calwerstr. 7

72076 Tübingen

Tel.: ++ 498/70 71/2982211

Fax: ++49/7071/2982211

markus.hahn@med.uni-

tuebingen.de

License terms

\section{Abstract}

$\nabla$

Purpose: The aim of this study was to evaluate the quality standard of the nationwide breast ultrasound training program of the German Society of Ultrasound in Medicine (DEGUM) through objective parameters.

Materials and Methods: 10 quality criteria, based on the recommendations of The National Association of Statutory Health Insurance Physicians (KBV), were defined for this study. All training units of the DEGUM received a questionnaire. The questionnaires and training material were analyzed.

Results: All units met the required criteria pertaining to the trainer's qualification, duration per training course and the maximum number of participants per ultrasound machine. Only 1 course did not fulfill the required $50 \%$ practical training time. The requirements to participate in the graduate course (200 self-made and documented cases) were not clearly conceived and a defined training log could be improved.

Conclusion: DEGUM breast ultrasound training offers trainees a high level of education based on the requirements of the KBV. Despite the high quality of training, the content of course announcements could be improved and an official and structured educational index could be meaningful.

\section{Einleitung $\nabla$}

Die Mammasonografie ist eine der wichtigsten bildgebenden Verfahren der Brust, die in Diagnostik und Therapie zum Einsatz kommt.

Voraussetzung für eine qualitativ hochwertige Ultraschalldiagnostik ist ein qualifizierter Untersucher mit entsprechender gerätetechnischer

\section{Zusammenfassung \\ $\nabla$}

Ziel: Die Evaluation der DEGUM-Mammasonografiekurse nach objektivierbaren Kriterien war Ziel dieser Arbeit. Damit sollte die Qualität der Kurse überprüft werden, um eine flächendeckende Fort- und Weiterbildung auf hohem Niveau anzubieten.

Material und Methoden: 10 Qualitätskriterien, orientierend an den Vorgaben der KBV-Ultraschallvereinbarungen wurden als Qualitätsparameter definiert. Alle Kursleiter des Arbeitskreises Mammasonografie der DEGUM wurden angeschrieben. Dabei wurden die 10 definierten Qualitätskriterien überprüft.

Ergebnisse: Alle Kurse erfüllten die Voraussetzungen bezüglich der Qualität des Kursleiters, der Kursdauer und Unterrichtseinheiten sowie der Anzahl an Kursteilnehmern pro Ultraschallgerät. In 1 von 9 Kursen wurde die Zeit der praktischen Übungen, gefordert sind 50\%, unterschritten. Die Voraussetzungen für den Abschlusskurs (200 selbst durchgeführte und dokumentierte Fälle) sind in den Kursankündigungen zum Teil nicht klar definiert. Ein strukturierter Lehrkatalog fehlt. Schlussfolgerung: Die DEGUM-Mammasonografiekurse werden auf hohem Niveau angeboten und erfüllen zum größten Teil die Anforderungen der KBV. Trotz der hohen Qualität der DEGUMKurse sind Optimierungsoptionen im Bereich Kursankündigung und strukturierter Lehrkatalog möglich.

Ausstattung einschließlich kontinuierlicher Weiterbildung.

Anforderungen an den Untersucher betreffen die Kenntnis über die physikalischen Grundlagen des Ultraschalls, die Übertragung von der Anatomie zur Sonoanatomie im Bereich Mamma und Axilla, Kenntnis über Krankheitsbilder der Mamma, die Fähigkeit zur Erkennung und Interpreta- 
tion von Dignitätskriterien sowie Wissen über die standardisierten Dokumentationsvorgaben und Interventionserfahrung. Als Qualitätskriterium im Bereich Mammasonografie im Rahmen der Zertifizierung von Brustzentren fordern die Deutsche Krebsgesellschaft und die Deutsche Gesellschaft für Senologie vom Untersucher DEGUM I analoge Kenntnisse oder die Teilnahme an Grund-, Aufbau- und Abschlusskurs Mammasonografie (www.senologie.org).

Abhängig von der Erfahrung und Spezialisierung des Untersuchers (objektiviert durch Fallzahlen) und Zusatzkriterien, wie z.B. Ausführung minimalinvasiver Interventionen oder wissenschaftlicher Tätigkeit im Bereich Mammasonografie, kann die Einteilung in die DEGUM-Stufen I-III als Qualitätsparameter erfolgen (www.DEGUM.de).

Die DEGUM greift bei der Ausbildung die Vorgaben der Ultraschallvereinbarung entsprechend der KBV ( $\$ 135$ Abs. 2 SGB: Vereinbarung von Qualitätssicherungsmaßnahmen zur Ultraschalldiagnostik) auf (www.kbv.de).

Der Arbeitskreis Mammasonografie der DEGUM bietet deutschlandweit qualitätsgesicherte Mammasonografiekurse an. Die Kursleiter erfüllen die Mindestanforderung DEGUM-II-Kursleiter oder gehören der Stufe III an. Die Kurse müssen bei der DEGUM angezeigt und vom Arbeitskreis genehmigt werden.

Mit über 700 Mitgliedern ist der Arbeitskreis Mammasonografie der größte Arbeitskreis innerhalb der DEGUM. 2011 waren im Bereich Mammasonografie nach dem DEGUM-Stufenkonzept 400 Mitglieder Stufe-I-, 126 Mitglieder Stufe-II- und 16 Mitglieder Stufe-III-qualifiziert. Insgesamt waren 22 Kursleiter gemeldet von denen 16 die Stufe III und 6 die Stufe II hatten.

Leider langt dies noch nicht aus, um eine flächendeckende Versorgung im Bereich Mammasonografie zu ermöglichen, was die Notwendigkeit qualifizierter Kurse umso mehr verdeutlicht.

Ziel dieser Arbeit war es, die Kurse der DEGUM, Arbeitskreis Mammasonografie unter dem Gesichtspunkt der Qualitätssicherung und einheitlicher Konzepte strukturell nach objektivierbaren Parametern zu evaluieren.

Im Rahmen dieser Versorgungsforschungsstudie wurden als Qualitätsindikatoren die Anforderungen der Ultraschallvereinbarung zugrunde gelegt und überprüft.

\section{Material und Methode}

$\nabla$

Im April 2011 wurden alle Kursleiter der DEGUM angeschrieben. Alle erhielten einen Fragebogen zu ihren Kursen (Fragebogen bei den Autoren). Die Auswertungen bezogen sich auf die Kurse aus dem Jahr 2010. Als Einschlusskriterium mussten die Kurse als Grund-, Aufbau- oder Abschlusskurs entsprechend der KVVorgaben durchgeführt worden sein. Als objektivierbare Parameter wurden 10 Qualitätskriterien aus den KV-Ultraschall-Vereinbarungen ausgewählt. Folgende Parameter wurden dabei analysiert:

1. Qualifikation der Kursleiter

2. Mindestdauer der Kursausbildung ( $§ 135$ : mindestens 9 Monaten zwischen Grund- und Abschlusskurs)

3. Einhalten der geforderten Kurszeiten (§135: Grundkurs 16 Kursstunden, Aufbaukurs 16 Kursstunden, Abschlusskurs 12 Kursstunden)

4. Kursstundendauer ( $§ 135: 45$ Minuten)

5. Prozentueller Anteil der praktischen Übungen an der Gesamtkurszeit ( $§ 135: 50 \%$ praktische Übungen)

6. Inhalte der theoretischen und praktischen Kursangebote
7. Teilnehmerzahl pro Ultraschallgerät während der praktischen Übungen ( $§ 135$ : maximal 5 Teilnehmer pro Gruppe)

8. Anzahl Referenten, Tutoren und Teilnehmer

9. Durchführung einer Prüfung im Abschlusskurs

10. Erfüllung der Teilnehmervoraussetzungen zum Abschlusskurs

Der Fragebogen bezog sich inhaltlich auf die oben genannten Qualitätsindikatoren sowie Anzahl der Mammasonografiekurse pro Jahr inklusive Angabe der Teilnehmerzahl, der Prüfungsunterlagen und des Zertifikats aus dem Jahr 2010.

Die Analyse der Kurszeiten bezieht sich in dieser Auswertung auf die jeweiligen Aufbaukurse.

\section{Ergebnisse \\ $\nabla$}

Zum Zeitpunkt der Umfrage waren bei der DEGUM 19 zertifizierte Kursleiter für das Fachgebiet Mammasonografie gemeldet.

Insgesamt konnten die Angaben von 9 Kursleitern ausgewertet werden.

3 Kursleiter boten zum Umfragezeitraum 2010 keine Kurse an. 2 Kursleiter boten 2010 Refresherkurse an, die nicht die Voraussetzung für die Umfrage erfüllen (Ausschluss). 3 Kursleiter boten keine eigenen Kurse an, sondern waren als Referenten bei den Kursen anderer Kursleiter tätig. Von 2 Kursleitern konnte kein Dateneingang verzeichnet werden.

1. Qualifikation der Kursleiter:

Alle Kursleiter hatten die Qualifikation DEGUM-II oder DEGUM-III. Nach den Vorgaben der DEGUM mussten alle Kursleiter einen Probevortrag vor dem Arbeitskreis Mammasonografie über ein vorgegebenes Thema halten um ihre didaktische Qualifikation und die Qualität ihres Bildmaterials nachzuweisen. Weitere Anforderungen an Kursleiter sind Kenntnisse in den Vorgaben der KV-Ausbildung sowie Fähigkeit in der Organisation einer Zweitagesveranstaltung mit Bereitstellung von ausreichend Technischer Hilfsmittel (USGeräte, Projektoren usw.). Bei der Mehrheit der Kurse waren 4 oder mehr Kursleiter als Referenten oder Tutoren tätig (๑ Tab. 1).

2. Mindestdauer der Ausbildung:

Gefordert wird ein Mindestabstand zwischen Grund- und Abschlusskurs von 9 Monaten. Die Mindestdauer zwischen Grund- und Abschlusskurs wurde in keinem Kursprogramm unterschritten. Wenn Teilnehmer alle 3 Kursabschnitte absolvierten, so konnten diese bei unterschiedlichen Kursleitern stattfinden.

8 der evaluierten Kursprogramme boten Grund-, Aufbau- und Abschlusskurs parallel an. Dabei konnten sich die Kursteilnehmer mit unterschiedlichen Vorkenntnissen die Vorträge nach ihrem Wissenstand in Modulen aussuchen bzw. je nach Kursniveau zugewiesen werden. Es wurde jeweils die Teilnahme an nur einem Kurs durch das Zertifikat bestätigt. Eine Zertifikatsausstellung über den Besuch mehrerer Kurse während eines 2-Tages-Kurs war durch keinen Kursleiter erfolgt.

3. Eine Unterschreitung der geforderten Pflichtstunden war in keinem Kurs erfolgt. Bei 3 Kursen wurde die vorgeschriebene Stundenzahl überschritten.

4. Nach Vorgaben der KV dauert eine Kursstunde 45 Minuten. Der Grundkurs umfasst $16 \mathrm{~h}$ an 2 Tagen, der Aufbaukurs $16 \mathrm{~h}$ an 2 Tagen, der Abschlusskurs $12 \mathrm{~h}$ an 2 Tagen: dies bedeutet eine konkrete Unterrichtszeit von 12 Zeitstunden für Grundund Abschlusskurs und 9 Zeitstunden im Abschlusskurs. Die- 
Tab. 1 Anzahl an Referenten, Tutoren und Seminarleitern pro Teilnehmer abhängig vom jeweiligen Kurs.

\begin{tabular}{|c|c|c|c|c|c|c|}
\hline Kurs & Anzahl Teilnehmer & $\begin{array}{l}\text { Anzahl Referenten } \\
\text { und Tutoren }\end{array}$ & $\begin{array}{l}\text { Anzahl der } \\
\text { Seminarleiter } \\
\text { (DEGUM-II und -III) }\end{array}$ & Betreuungsquotient & $\begin{array}{l}\text { Anzahl } \\
\text { Ultraschallgeräte } \\
\text { im Kurs }\end{array}$ & $\begin{array}{l}\text { Teilnehmer pro } \\
\text { Ultraschallgerät }\end{array}$ \\
\hline 1 & 60 & 15 & 5 & 0,25 & 5 & 5 \\
\hline 2 & keine Angabe & 15 & 5 & & k. A. & k. A. \\
\hline 3 & $60-70$ & 25 & 4 & 0,36 & 6 & 5 \\
\hline 4 & $180-200$ & 12 & 4 & 0,06 & k. A. & 5 \\
\hline 5 & 50 & 20 & 3 & 0,4 & 5 & 5 \\
\hline 6 & 70 & keine Angabe & 1 & & 5 & 5 \\
\hline 7 & 70 & 12 & 4 & 0,17 & 4 & 5 \\
\hline 8 & $15-20$ & 2 & 1 & 0,1 & 2 & 5 \\
\hline 9 & 250 & 45 & 4 & 0,14 & k. A. & k. A. \\
\hline
\end{tabular}

\begin{tabular}{|lll}
\hline Tab.2 & Prozentualer Anteil der praktischen Übungen eines Kurses. \\
\hline Kurs & $\begin{array}{l}\text { Anteil praktische } \\
\text { Übungen gesamt in \% }\end{array}$ & $\begin{array}{l}\text { Anteil praktische Übungen direkt am } \\
\text { Ultraschallgerät in \% (bezogen auf die } \\
\text { Gesamtzeit der praktischen Übungen) }\end{array}$ \\
\hline 1 & $50 \%$ & $35 \%$ \\
\hline 2 & $50 \%$ & $60 \%$ \\
\hline 3 & $50 \%$ & $50 \%$ \\
\hline 4 & $60 \%$ & $75 \%$ \\
\hline 5 & $50 \%$ & $100 \%$ \\
\hline 6 & $40 \%$ & $75 \%$ \\
\hline 7 & $50 \%$ & $75 \%$ \\
\hline 8 & $50 \%$ & $100 \%$ \\
\hline 9 & $60 \%$ & $25 \%$ (multimodale Bildgebung: $60 \%)$ \\
\hline
\end{tabular}

se Zeitvorgaben der Gesamtkursdauer wurden in allen Kursen erfüllt. Die Kurseinheiten betrugen in 5 Kursen $30-$ 45 Minuten, in 4 Kursen 45 - 60 Minuten.

5. Der prozentuale Anteil der praktischen Übungen am Gesamtkurs muss seitens der KV 50\% betragen. Innerhalb des Kurssystems werden praktische Übungen am Ultraschallgerät an der Probandin oder am Phantom durchgeführt. Weitere praktische Übungen betreffen die Bildinterpretation i.d. R. durch Fallbeispiele mit Bildertraining. In 6 Kursen wurden in 50\% der Kursdauer praktische Übungen durchgeführt, in 2 Kursen waren es 60\%. Den Anteil der Übungen direkt am Ultraschallgerät mit Probandin oder am Simulationsmodel zeigt - Tab. 2.

In einem Kurs wurden die Anforderung von 50\% praktischer Übungen an der Gesamtkursdauer unterschritten. Dieser Kurs beinhaltet die multimodale Mammadiagnostik und damit auch Praxisanteile Mammografie und MRT, die hier nicht gewertet wurden. In einem weiteren Kurs wurde die Rate von 50\% knapp unterschritten (Kurs 6: 40\%, dafür aber in der verbleibenden Praxiszeit ein sehr hoher Anteil an Übungen an der Probandin und/oder am Phantom angeboten).

6. Zusammenfassung der Inhalte der theoretischen und praktischen Kursangeboten (übernommen aus den jeweiligen Kursprogrammen):

Inhalt der praktischen Übungen:

Ultraschalluntersuchungen an Probandinnen, Übungen zu minimalinvasiven Interventionen am Phantom, US-Beurteilungskriterien im bewegten Bild, US-Beurteilungskriterien an Key-Pictures, Wissenskontrolle, interaktive Befundbeschreibung, Großbildvideodemonstration von Ultraschallun- tersuchungen an Probandinnen sowie von minimalinvasiven Interventionen, klinische Untersuchung der Brust, Fallvorstellung, Ultraschallquiz.

Theoretische Lehrinhalte mit direktem Bezug zur Mammasonografie:

DEGUM-Stufenkonzept und Ultraschallvereinbarung mit der $\mathrm{KV}$, benigne Befunde, maligne Befunde, Schallphysik und Gerätetechnik, Sonoanatomie, Untersuchungstechnik, Befunddokumentation; Sonografie bei Rekonstruktion/Implantat; Präparatesonografie; Axillasonografie, 3D-Sonografie, Dopplersonografie, Elastografie, Interventionen, Nachsorge, Duktoskopie und Duktsonografie, Geräteeinstellung, Stellenwert der Sonografie, komplementäre Mammadiagnostik, problem- und lösungsorientiertes Arbeiten in der Mammasonografie, Qualitätssicherung, Tumornachsorge, komplementäre Diagnostik, Artefakte und optische Täuschungen, Ultraschall im Mammografiescreening

Theoretische Lehrinhalte ohne direkten Bezug zur Mammasonografie:

S3-Leitlinie und MRT, Benchmarking beim Mammakarzinom, Indikationen der MG/MRT, Interventionen MG/MRT, erblicher Brustkrebs, Sport und Brustkrebs, moderne Strahlentherapie, psychologische Betreuung von Frauen mit Mammakarzinom.

7. Teilnehmerzahl pro Ultraschallgerät während der praktischen Übungen:

In 7 von 9 Kursen wurden maximal 5 Kursteilnehmer pro Ultraschallgerät während der praktischen Übungen eingeplant. Von 2 Kursen konnten keine Angaben erhoben werden.

8. Anzahl der Referenten, Tutoren und Teilnehmer:

Um die Betreuungsqualität der praktischen Übungen abzubilden, wurde ein Quotient zwischen Anzahl der Lehrenden und Kursteilnehmern gebildet ( $\bullet$ Tab. 1). Dieser Betreuungsquotient ist bisher rein deskriptiv zu verstehen.

9. Durchführung einer Abschlussprüfung:

In allen 9 Kursen wurde im Rahmen des Abschlusskurses eine Wissensüberprüfung durchgeführt. In 5 Programmen wurde bereits auf die Abschlussprüfung als Bestandteil des Abschlusskurses hingewiesen. 5 Kurse führten die Prüfung kombiniert praktisch-theoretisch durch. 6 Kurse führten zum Abschluss für alle Teilnehmer eine Wissenüberprüfung an Fallbeispielen unabhängig davon durch, ob es sich um Grund-, Aufbau- oder Abschlusskurs handelt.

10. Erfüllung der Teilnehmervoraussetzung für die Abschlussprüfung: 
An der Abschlussprüfung als Bestandteil des Abschlusskurses kann man nach Vorgabe der KV §135 nur teilnehmen, wenn man 200 selbstdokumentierte Fälle vorlegt. Der Nachweis von 200 selbstdokumentierten Fällen als Voraussetzung zur Prüfungszulassung wurde in keiner Kursankündigung erwähnt. In 2 Kursen wurde die Durchführung von 200 Fällen überprüft. 2 Kurse bestätigten die korrekte Durchführung und Dokumentation von den 200 vorgelegten Fällen. Sieben Kurse machten hierzu keine Angaben in den Ankündigungsflyern. Die Abschlusskursteilnehmer wurden aber bei der Kursanmeldung über den Nachweis 200 selbstdokumentierter Fälle informiert.

\section{Diskussion \\ $\nabla$}

In den vergangenen 2 Jahrzehnten ist die Mammasonografie elementarer Bestandteil der diagnostischen Kette bei Erkrankungen der Brust geworden. Der Ultraschall stellt heute „das Auge des Senologen“ dar, da diese Technik für die Patientin im Vergleich zur Mammografie ohne Strahlenbelastung einhergeht, im Vergleich zur MRT kostengünstig und ohne Kontrastmittelbelastung durchführbar ist. Dabei bietet die Mammasonografie eine hohe Sensitivität (98,4\%) bei guter Spezifität (67,8\%) [1]. Der Mammaultraschall ist sowohl in der Früherkennung als auch bei der Abklärung symptomatischer Patientinnen einsetzbar. Weitere Indikationsspektren betreffen die bildgebende Zielsteuerung minimalinvasiver Interventionen, die intraoperative Bildgebung sowie den therapeutischen Einsatz (High Intensity Focused Ultrasound - HIFU).

Um die Mammasonografie fachgerecht durchzuführen, ist eine hochwertige Ausbildung mit kontinuierlicher Weiterbildung notwendig.

Die Deutsche Gesellschaft für Ultraschall in der Medizin (DEGUM) bietet durch den Arbeitskreis Mammasonografie ein Kurssystem an, bei dem die Qualität der Kursleiter sowie der Seminare klaren Qualitätsvorgaben entsprechen müssen. Diese Qualitätsvorgaben orientieren sich zum einen am Stufenkonzept der DEGUM einschließlich Kursleiterstatus zum anderen werden darin die Vorgaben der KV berücksichtigt.

In der vorliegenden Arbeit wurden die Kurse des Arbeitskreises Mammasonografie anhand von objektivierbaren Qualitätsparametern evaluiert.

Dabei konnte gezeigt werden, dass alle Kurse von qualifizierten DEGUM-Kursleitern geleitet werden, die über jahrelange Erfahrung in der Mammasonografie und über entsprechende didaktische Fähigkeiten verfügen. Als Kursleiter können sich Untersucher der DEGUM-Stufe-II bewerben. Mitglieder der Stufe III erfüllen gleichzeitig die Voraussetzungen als Kursleiter. Sie müssen neben dieser Mindestqualifikation über ausreichend Bildmaterial verfügen, Kenntnisse und Fähigkeiten in der Durchführung von Weiterbildungskursen besitzen und ihre didaktischen Fähigkeiten durch eine Prüfung vor dem Arbeitskreis nachweisen.

Dieser Qualitätsnachweis für Kursleiter sollte heute grundsätzlich gefordert werden, da aufgrund wirtschaftlicher Bedingungen in vielen Kliniken ein Defizit an qualifizierten Ultraschallausbildern und Ultraschalldiagnostikern für die Supervision der praktischen Ausbildung sowie der Kursleitung entstanden ist [2]. Diese Situation stellt aktuell eine Herausforderung insgesamt im deutschen Gesundheitssystem dar.

Eine Analyse zur Qualitätssicherung von Ultraschallkursen in der ärztlichen Fort- und Weiterbildung von Hofer et al. [3] belegte vor 10 Jahren erhebliche Defizite im didaktischen Aufbau der damals untersuchten Ultraschallkurse. Besonders kritisiert wurden hohe Anteile von Frontalunterricht, unstrukturierte Praxisübungen in großen Gruppen mit hohen Abwesenheitsquoten der Teilnehmer und zu lange Kurseinheiten. Als Konsequenzen wurde insbesondere eine praxisnähere Ausbildung sowie didaktische Schulung der Ausbilder gezogen.

Schlussfolgerung aus der Arbeit von Hofer waren u. a.

- ein alternierendes Kursdesign mit häufigem Wechsel zwischen kurzen Theorie- und längeren Praxismodulen,

- längere Praxismodule mit höchstmöglicher Teilnehmeraktivität unter intensiver, strukturierter Anleitung in Kleingruppen ( $\leq 5$ Teilnehmer pro Gruppe, Arbeitsplatz und Ausbilder),

- kurze Theoriemodule, deren Sachvorträge durch dosierten Einsatz moderner Medien visuell veranschaulicht werden,

- professionelle didaktische Schulung von Kursleitern und Tutoren,

- die Durchführung von Lernzielkontrollen zur Überprüfung praktischer Handlungskompetenz als Voraussetzung für die Zertifikatvergabe.

Wie die vorgelegte Arbeit zeigt, werden viele der von Hofer geforderten Zielkriterien bereits jetzt schon in den Kursen des Arbeitskreises Mammasonografie umgesetzt. Ein monotoner Frontalunterricht findet in keinem der Kurse statt. Der Wechsel zwischen theoretischen und praktischen Einheiten ist bei den Kursen der DEGUM mittlerweile Standard. Mit einer Ausnahme erfüllten alle Kursangebote die Bedingung eines 50\% Praxisanteils. Dieser Praxisteil stellt jedoch für die Organisation der Kurse eine logistische und finanzielle Herausforderung dar. Zum einen muss die Gerätetechnik (Ultraschallgeräte, Biopsiegeräte, Phantome usw.) zur Verfügung gestellt werden, zum anderen müssen ausreichend Probandinnen für die Übungen ausgewählt und anwesend sein ( $\bullet$ Abb.1). Einige Kurse ermöglichen die praktischen Übungen an Probandinnen mit pathologischen Befunden, um den Lerneffekt noch zu optimieren. Die praktischen Übungen am Ultraschallgerät werden in Kleingruppen mit max. 5 Teilnehmern durchgeführt, während andere praktische Übungsteile, wie z. B. BIRADS-Training an Fallbeispielen in z.T. größeren Gruppen stattfinden. Die didaktischen Fähigkeiten der Tutoren werden z.T. bereits in den Evaluationsbogen der Kurse überprüft. In der überwiegenden Mehrheit der Kurse beträgt eine Kurseinheit 45 Minuten. In vier der Kurse wird diese Zeit unterschritten, was di-

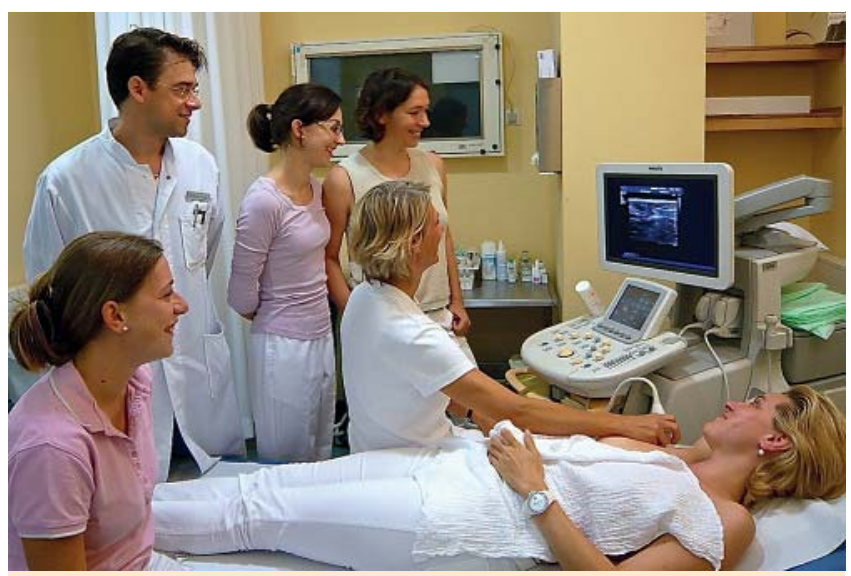

Abb.1 Optimale Ultraschallausbildungsbedingungen im praktischen Kursteil (1 Probandin, 1 Tutor (hier Kursleiter selber) und 4 Kursteilnehmern). 
daktisch als vorteilhaft eingestuft wird. Eine Wissensüberprüfung findet in allen Abschlusskursen statt. In 2/3 der untersuchten Kurse wurde eine Wissensüberprüfung in allen Kursabschnitten durchgeführt.

Einen einheitliche Evaluationsbogen, der eine Rückmeldung durch die Kursteilnehmer ermöglichen würde, gibt es bisher für die Kurse des Arbeitkreises Mammasonografie nicht. Eine Evaluation durch die Kursteilnehmer könnte zur kontinuierlichen Optimierung der Kurse aus Sicht der Teilnehmer beitragen. Dabei sollte eine Evaluation nicht nur die inhaltlichen Aspekte der Kurse, sondern auch die Struktur (z.B. Anzahl der Teilnehmer pro Tutor und Schallgerät bei den praktischen Übungen) untersuchen.

Im Rahmen dieser Arbeit konnten Optimierungspunkte für die Kurse herausgearbeitet werden. Die Kursankündigung durch den Kursflyer sollte alle notwendigen Informationen erhalten, die auch den Qualitätsvorgaben der KV entsprechen. Vor allem ist auf die Vorlage der 200 Fälle für Abschlusskursteilnehmer hinzuweisen. In den Kursankündigungen der DEGUM-Homepage ist dieser Hinweis aber abgebildet. Nach unserer Einschätzung ist zum Teil auch die parallele Durchführung der Grund-, Aufbauund Abschlusskurse für die Teilnehmer nicht verständlich in den Kursankündigungen dargestellt. Es sollte erkennbar sein, dass die Teilnahme nur an einem Kursteil pro 2 Unterrichtstage möglich ist, und zwischen Grund- und Abschlusskurs 9 Monate Zeit (Vorgabe der KBV) liegen müssen. Dies sollte zukünftig stärker bei der Anmeldung und bei der Kursdurchführung berücksichtigt werden. Eine von der DEGUM vorgegebene Eigenevaluation der Kurse mit einheitlichen und vergleichbaren Qualitätsindikatoren wäre sinnvoll. Um die Lehrinhalte klar zu definieren und in den einzelnen Kursmodulen transparent zu machen, wäre ein Lehrkatalog oder Logbuch zu empfehlen.

Im Jahre 2012 wurden nach offiziellen Angaben der DEGUM 2731 Kolleginnen und Kollegen in den Mammsonografiekursen der DEGUM ausgebildet. Dabei wird das Kursangebot sowohl von Kolleginnen und Kollegen in der Weiterbildung als auch von erfahrenen Kolleginnen und Kollegen aus der Niederlassung und Klinik wahrgenommen. Diese große Zahl zeigt das hohe Interesse an der Ultraschallausbildung.

Die Kosten für die Kursteilnahme für einen 2 Tageskurs betragen durchschnittlich $450 €$. In die Kostenkalkulation gehen u.a. die Kosten für die Referenten, Tutoren, Probandinnen, Ultraschallgeräte, Räumlichkeiten und Catering mit ein. Ob sich dies bei rückläufiger Tendenz der Sponsoren weiter halten lassen wird, ist fraglich. Die Kooperation mit Geräteherstellern ist aber notwendig, um die neuesten Techniken und Möglichkeiten dem Kursteilnehmer darstellen zu können. Die Neufassung der Qualitätssicherungsrichtlinien der KBV wird die Regelungen zur fachlichen Qualifikation des Arztes und zur apparativen Ausstattung ändern - dies gilt momentan für die vertragsärztliche Tätigkeit, wird sich aber auch auf die Klinikärzte auswirken. Es ist ein Trend, der sich zugunsten der Prozess- und Ergebnisqualität auch in der Ausbildung Mammasonografie fortsetzen wird, wie beispielsweise die Qualitätsoffensive Ultraschall zeigt [4].

Ob sich moderne internetbasierte Lern- und Lehrinstrumente durchsetzen werden und Kurse in Ihrem Ausbildungsauftrag unterstützen können, wird die Zukunft zeigen [5]. Die sonografischen Fähigkeiten von Untersuchern können evtl. durch eine simulatorgestütze Ausbildung verbessert werden. Der Arbeitskreis Mammasonografie nutzt bisher keine digitalen oder internetbasierten Lern- oder Lehrinstrumente. Ob derartige Instrumente in der Zukunft integriert werden, wie dies zum Beispiel bei der Pränatalmedizin bereits der Fall ist, wird sich zeigen müssen. Aus Sicht der Autoren wäre ein solches Konzept zu unterstützen. Der Effekt solcher Methoden wurde für andere Organe in der Ultraschalldiagnostik bereits nachgewiesen [6].

Die hohe Qualität der Kurse spiegelt sich auch durch die Abbildung der DEGUM-Kriterien in der S3-Leitlinie zur Therapie und Diagnostik des Mammakarzinoms und im Erhebungsbogen zur Zertifizierung von Brustzentren nach DGS und DKG wieder.

\section{Zusammenfassung}

1. Unsere Auswertungen zeigen, dass die DEGUM-Kurse Mammasonografie, bis auf eine Ausnahme in einem Kriterium, alle Voraussetzungen der neuen KBV-Ultraschallvereinbarung erfüllen.

2. Der geforderte hohe Prozentsatz an praktischen Übungen stellt eine logistische und finanzielle Herausforderung für die Organisation der Kurse dar.

3. Das Kursangebot der DEGUM bildet das komplette Spektrum der Mammasonografie inklusive Interventionen sinnvoll ab. Ein definierter strukturierter Lehrkatalog existiert bisher noch nicht.

4. Für die Zukunft wäre es wünschenswert, grundsätzlich die Kurse des Arbeitskreises Mammasonografie der DEGUM durch die Teilnehmer evaluieren zu lassen, wobei die Erarbeitung und Verwendung eines einheitlichen Bewertungsbogens sinnvoll wäre.

\section{Affiliations}

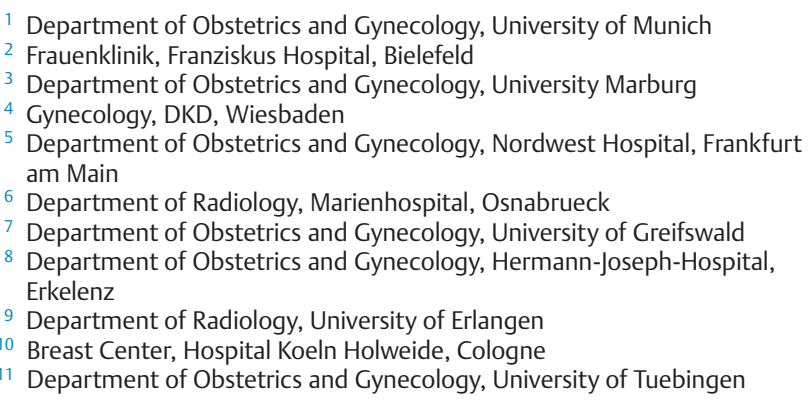

\section{Literatur}

1 Stavros AT, Thickman D, Rapp CL et al. Solid breast nodules: use of sonography to distinguish between benign and malignant lesions. Radiology 1995; 196: 123 - 134

2 Jaeger KA. Training and education in ultrasound. Ultraschall in der Medizin 2012; 33: 119-121

3 Hofer M, Mey N, Metten J et al. Quality control of sonography courses in advanced training of physicians: analysis of present status and potential for improvement. Ultraschall in der Medizin 2002; 23: 189-197

4 Merz E, Batzel A. Quality assurance in ultrasound diagnostics where are we at the end of 2009? Ultraschall in der Medizin 2009; 30: $525-527$

5 Grunewald M, Gebhard H, Wagner M et al. Quality improvement of resources in radiology on the internet. Fortschr Röntgenstr 2005; 177: $569-575$

6 Staboulidou I, Freitag U, Marquardt R et al. Quality assured ultrasound simulation training for the detection of fetal malformations - can a training benefit be evidenced? Zeitschrift fur Geburtshilfe und Neonatologie 2006; 210: 135-140 